\title{
Comité de ética hospitalaria: una instancia ineludible para el nutricionista clínico
}

\author{
Hospital ethics committee: An unavoidable authority \\ for the clinical nutritionist \\ Comitê de ética hospitalar: uma instância inevitável para o nutricionista clínico
}

\author{
Iván Pérez ${ }^{1 *}$
}

Recibido: 29 de marzo de 2021. Aceptado para publicación: 22 de julio de 2021. https://doi.org/10.35454/rncm.v4supl1.321.

\section{Resumen}

El acelerado avance de la medicina y los cambios en la relación médico-paciente han significado un aumento en la complejidad de las decisiones clínicas, lo que da origen a problemas ético/clínicos; es decir, aquellos en los que están involucrados conflictos de valor. Estos consisten en una dificultad para especificar lo que debe hacerse y no solo lo que puede hacerse o frecuentemente se hace. En este contexto surgen los comités de ética hospitalaria, que son órganos consultivos de carácter multidisciplinario, cuyo objetivo es ayudar al equipo sanitario a tomar las mejores decisiones terapéuticas en casos particulares. Cuando se consultan, estos comités deliberan en torno al problema ético-clínico, utilizando una metodología y una sistematización del análisis, teniendo como referencia las distintas corrientes de fundamentación ética. La interconsulta ética individual es una opción complementaria a los comités de ética. Los motivos más frecuentes de consulta ética son la adecuación del esfuerzo terapéutico y los conflictos relacionados con el ejercicio de la autonomía del paciente. Ambos aspectos están involucrados en los dilemas éticos, asociados con la nutrición e hidratación administrada artificialmente en distintos contextos clínicos, como en la anorexia nerviosa, la demencia avanzada, el paciente terminal en cuidados paliativos, en estados de mínima consciencia y en el estado
\end{abstract}

\section{Abstract}

The accelerated progress of medicine and the changes in the patient-physician relationship have resulted in increasingly complex clinical decisions, giving rise to ethical-clinical problems, that is, those in which value conflicts are involved. These consist of difficulty in specifying what must be done and not only what can be done or is frequently done. Hospital ethics committees emerge in this context as multidisciplinary advisory bodies whose objective is to help the healthcare team make the best therapeutic decisions in particular cases. When consulted, the committees deliberate on the ethical-clinical problem using a methodology and a systematic analysis, within the framework of the different trends of ethical foundation. Individual ethical consultation is a complementary option to ethics committees. The most frequent reasons for ethical consultation are the adequacy of the therapeutic effort and conflicts related to the exercise of patient autonomy. Both aspects are involved in the ethical dilemmas associated with artificially administered nutrition and hydration in different clinical contexts, such as anorexia nervosa, advanced dementia, terminally ill patients in palliative care, states of minimal consciousness and chronic persistent vegetative state. Therefore, clinical nutritionists may consider consulting

\section{Resumo}

O avanço acelerado da medicina e as mudanças na relação médico-paciente têm significado um aumento na complexidade das decisões clínicas, gerando problemas ético-clínicos, ou seja, aqueles em que estão envolvidos conflitos de valores. Trata-se de uma dificuldade em especificar o que deve ser feito e não apenas o que pode ser feito ou freqüentemente é feito. Nesse contexto, surgem os comitês de ética hospitalar, que são órgãos consultivos multidisciplinares que têm como objetivo auxiliar a equipe de saúde na melhor tomada de decisões terapêuticas em casos particulares. Quando consultados, os comitês deliberam sobre a problemática ético-clínica a partir de uma metodologia e de uma sistematização da análise, tomando como referência as diferentes correntes da fundamentação ética. A interconsulta ética individual é uma opção complementar aos comitês de ética. Os motivos mais frequentes para a consulta ética são a adequação do esforço terapêutico e os conflitos relacionados ao exercício da autonomia do paciente. Ambos os aspectos estão envolvidos nos dilemas éticos associados à nutrição e hidratação administrada artificialmente em diferentes contextos clínicos, como anorexia nervosa, demência avançada, pacientes terminais em cuidados paliativos, estados de consciência mínima e estado vegetativo crônico persistente. Portanto, 
vegetativo persistente crónico; por tanto, el nutricionista clínico puede considerar la consulta al comité de ética hospitalario cuando enfrente decisiones difíciles de su especialidad.

the hospital ethics committee when faced with difficult decisions in their specialty.

Keywords: Ethics committee; Ethics; Nutrition. o nutricionista clínico pode considerar consultar o comitê de ética do hospital ao se deparar com decisões difíceis em sua especialidade.

Palabras clave: comités de ética, ética, nutrición.

Facultad de Medicina, Pontificia Universidad Católica de Chile, Santiago, Chile.

\section{INTRODUCCIÓN}

La toma de decisiones en medicina clínica plantea crecientes desafíos de orden técnico y también moral, lo que se explica por la rápida evolución que han tenido dos aspectos relevantes de la actividad sanitaria en las últimas 6 o 7 décadas. En primer lugar, asistimos a un cambio en la relación médico-paciente, tradicionalmente paternalista y vertical, que ha dado paso a un progresivo reconocimiento de la autonomía del enfermo, su participación en la toma de decisiones y la necesidad de considerar sus intereses y preferencias, no siempre concordantes con las recomendaciones técnicas del médico ${ }^{(1)}$. Surge así un modelo de relación clínica de tipo participativo-informativo, que reconoce en el paciente (o en quien lo represente en casos de incapacidad para decidir) la posibilidad de aceptar o rechazar una propuesta terapéutica.

En segundo lugar, y no por ello menos importante, se ha producido un acelerado avance del conocimiento y del desarrollo tecnológico en la medicina, lo que ha permitido incorporar a la atención rutinaria de salud intervenciones de altísima complejidad y costos, logrando el control de muchas enfermedades, la recuperación de pacientes gravemente enfermos y el aumento de la expectativa de vida. Estos nuevos procedimientos diagnósticos y terapéuticos se aplican en todas las etapas del ciclo vital, dando origen a nuevas situaciones, como es el caso de pacientes que logran sobrevivir a eventos críticos, aunque con graves secuelas, que afectan su calidad de vida y demandan cuidados especiales a sus familias y exigencias a la sociedad. Entre estos cuidados y tratamientos se encuentran aquellos relacionados con la alimentación que, aunque corresponde a una necesidad básica para sostener la vida del ser humano, en ciertos contextos clínicos plantea dificultades respecto a la obligatoriedad

de su administración, en especial, cuando se requiere de vías artificiales.

Una resultante inesperada y, en cierto modo, paradójica a estos notables avances ha sido un visible descontento de los pacientes con esta medicina altamente especializada y tecnológica, la que se percibe como deshumanizada y poco centrada en la persona ${ }^{(2)}$. En oportunidades, la relación profesional sanitario-paciente se desarrolla como una transacción comercial, en la que un cliente solicita algunas prestaciones o servicios a un proveedor, en una relación fundada más en los derechos y deberes de quienes interactúan, que en la confianza. Es así como el descontento, la desconfianza y una progresiva «judicialización» de la medicina explican la tendencia a practicar el arte médico «a la defensiva», lo que en cierto punto desvirtúa los fines primarios de la profesión.

Es en este contexto histórico y sociocultural en el que surgen los comités de ética hospitalarios (CEH), que pasan a constituirse, en instancias consultivas relevantes, para apoyar a los clínicos y a los pacientes en la toma de decisiones complejas, que involucran aspectos valóricos. A ellos también puede recurrir el nutricionista clínico cuando se enfrenta a problemas ético-clínicos de su especialidad. Este artículo tiene como objetivos mostrar qué es un CEH y cuáles son los dilemas del área nutricional que deberían motivar una consulta a dicho comité.

\section{COMITÉS DE ÉTICA HOSPITALARIA}

\section{Sus comienzos y situación actual}

Los comités de ética hospitalaria (hospital/clinical ethics committee) surgen en la segunda mitad del siglo XX en Estados Unidos, siendo probablemente uno de sus primeros referentes el Comité de Seattle, que se 
constituyó en 1960 para decidir qué pacientes podían acceder al revolucionario tratamiento de hemodiálisis, considerando el gran número de candidatos con insuficiencia renal terminal y la escasez del recurso. Por dicho motivo, este se conoció como el comité de la vida o la muerte. Luego hubo otros casos emblemáticos, como el de Karen Ann Quinlan en 1976 o los casos denominados «Baby Doe» y «Baby Jane Doe» en 1982 y 1983, que pusieron en evidencia la complejidad de las decisiones médicas ante el surgimiento de nuevas tecnologías médicas, como la ventilación mecánica invasiva, las unidades de paciente crítico o los primeros trasplantes de órganos.

Algunos de estos casos llegaron a las cortes de justicia y a los medios de comunicación social, lo que causó gran impacto en la opinión pública. Esto fue otro motivo para crear comités de ética en los hospitales, que ayudaran a resolver estas complejas situaciones, en particular, cuando ocurrían en pacientes con incapacidad mental, inconscientes o en recién nacidos gravemente enfermos.

Desde esa época, el surgimiento de los $\mathrm{CEH}$ ha sido exponencial. En 1983, solo el $1 \%$ de los hospitales norteamericanos contaba con su comité, cifra que aumentó a 60 \% en 1989 y a más de $93 \%$ en 1999. La Asociación Médica Británica creó sus propios comités en 1981. En España, Chile y otros países latinoamericanos, los $\mathrm{CEH}$ se institucionalizaron a partir de 1990. En la actualidad, todos los hospitales acreditados por la Joint Commission on the Accreditation of Health-Care Organizations, tanto en Estados Unidos como en el resto del mundo, deben contar con su propio mecanismo de resolución de consultas éticas. Asimismo, la existencia de $\mathrm{CEH}$ ha pasado a ser una obligación legal, como ocurre en Chile, donde su constitución y funciones están establecidos en la Ley de derechos y deberes de las personas en su atención de salud (Ley 20584), y su respectivo Reglamento ${ }^{(3)}$.

\section{Definición, conformación y ámbito de acción de los CEH}

Los CEH son grupos interdisciplinarios, que se ocupan de la docencia, investigación y consulta asociadas con los dilemas éticos, que surgen durante la práctica de la medicina hospitalaria ${ }^{(4)}$. Tienen tres ámbitos generales de acción: atender a la formación bioética tanto entre los propios miembros del comité, como en el conjunto del hospital y hacia la comunidad; desarrollar políticas y orientaciones generales para la institución; y asesorar al personal sanitario en la toma de decisiones, en las que vienen implicados conflictos de valor ${ }^{(5)}$.

La importancia relativa a cada una de estas funciones es muy diferente según el país, el tipo de institución y las necesidades temporales de cada hospital. De estas tres funciones, la que mejor responde a las necesidades clínicas de médicos y pacientes es el rol consultivo que los comités cumplen en los hospitales, lo que constituye una instancia formal de asesoría en la toma de decisiones clínicas en las que se confrontan principios y valores.

En 1981, Bernard Lo definió un problema ético clínico como una dificultad en la toma de decisiones frente a un paciente, en cuya resolución es necesario referirse a valores o principios que especifiquen lo que debe ser hecho, en oposición a lo que simplemente puede ser hecho o frecuentemente se hace ${ }^{(6)}$. Por tanto, el ámbito primario de acción de un $\mathrm{CEH}$ es la dimensión ética del actuar médico (la bondad o maldad de las acciones) y no su dimensión técnica, la que en otras instancias puede evaluarse utilizando criterios de eficacia y eficiencia, en referencia al conocimiento prevalente (lex artis medicae) y a la información que aporta la medicina basada en la evidencia.

Para no desvirtuar los fines que le son propios, es relevante mencionar qué funciones no debiera realizar un $\mathrm{CEH}$. En general, estos comités no prestan amparo jurídico directo a quienes trabajan en los hospitales, aunque sí lo hacen indirectamente por el efecto que tiene la deliberación ética formal de un caso frente al paciente, la familia o la justicia ${ }^{(7)}$; tampoco se constituyen en instancias de juicio o sanción al personal sanitario; no realizan análisis económicos, aunque este aspecto tenga repercusiones éticas; finalmente, los $\mathrm{CEH}$ no debieran asumir tareas propias de los comités de ética científica o de investigación clínica, los que tienen su propio marco regulatorio de acción y cuyas recomendaciones tienen carácter vinculante para los investigadores ${ }^{(8)}$, a diferencia de los CEH cuyas recomendaciones pueden calificarse como sugerencias calificadas, que apoyan pero no reemplazan la responsabilidad de los médicos tratantes.

Con respecto a su conformación, la tendencia predominante ha sido que los $\mathrm{CEH}$ estén constituidos por profesionales clínicos (médicos, enfermeros, kinesiólogos, psicólogos, entre otros) con especial motivación y competencia en la ética médica, junto con un especialista en ética y un asesor legal. En algunos casos se incluye un asesor espiritual y un representante de la comunidad. Una revisión sistemática reciente de la evolución de los $\mathrm{CEH}$ en Estados Unidos, país que ha 
liderado su incorporación formal en el quehacer hospitalario, ha demostrado que la conformación de los comités ha cambiado en el tiempo, destacando la tendencia a aumentar el número de sus integrantes (15-16 en general) y la proporción de profesionales no médicos, predominantemente del área de la enfermería, así como de representantes de la comunidad ${ }^{(9)}$. Datos comparables se han reportado sobre la evolución de estos comités en el sistema de salud canadiense ${ }^{(10)}$.

Pese a estar ampliamente presentes en el sistema de atención de salud a nivel mundial, los $\mathrm{CEH}$ son escasamente consultados, considerando la multiplicidad de dilemas éticos que plantea la medicina actual, aunque esto podría cambiar. En Estados Unidos, el promedio de consultas anuales a los $\mathrm{CEH}$ aumentó de 7,2 a 19,5 en las últimas 4 décadas, aun cuando se evidenció una gran dispersión en las cifras, con algunos comités que fueron consultados más de 200 veces al año ${ }^{(9)}$. Esta constatación ha motivado el desarrollo de la interconsulta ética individual, como una modalidad alternativa a los comités de ética hospitalarios en la resolución de dilemas ético-clínicos, especialmente en unidades de paciente crítico ${ }^{(11)}$.

La mayor ventaja es la oportunidad de respuesta, considerando que la consulta se atiende por un experto sin necesidad de convocar a un comité, con eventual disponibilidad las 24 horas del día, si se organiza un sistema de turnos; la evaluación de los casos se puede hacer en las mismas unidades clínicas, lo que facilita el contacto directo con los médicos, pacientes y familiares. Incluso es una oportunidad para capacitar «en terreno» a los integrantes del equipo de salud en temas de la bioética aplicada a casos concretos. Esta mayor eficiencia tiene como principal debilidad que la opinión y sugerencias son dependientes de las cualidades personales del interconsultor; asimismo, se pierde la posibilidad de una deliberación multidisciplinaria, que es lo más propio de los $\mathrm{CEH}^{(12)}$. La propuesta actual es considerar ambas modalidades de consulta como complementarias y sinérgicas, para generar un sistema de consultoría mixto, que acoja oportunamente los requerimientos del equipo sanitario.

\section{Deliberación en los CEH}

Ya se ha mencionado que las decisiones complejas en la práctica médica conllevan aspectos técnicos y éticos. Se trata de alcanzar un grado de certeza técnica y moral antes de actuar, certezas que no son de tipo apodíctico, sino más bien el resultado de un juicio prudencial, que busca elegir la mejor opción para ese caso concreto. Se requiere conocer las circunstancias clínicas, considerar el conocimiento prevalente y reconocer los principios y valores en juego, teniendo como fin último la consecución del bien integral del paciente.

En la mayoría de los casos es el mismo médico tratante, especialmente cuando tiene mayor experiencia clínica, quien resuelve, de un modo casi intuitivo, las dificultades técnicas y éticas. Sin embargo, existen situaciones de mayor complejidad, que demandan un análisis metódico y fundamentado, tarea propia de los comités de ética hospitalarios. Para entender mejor el proceso que ocurre al interior de dichos comités es necesario distinguir tres niveles conceptuales: los modelos o tendencias bioéticas de referencia, los modelos de sistematización del análisis $y$, finalmente, las posibles metodologías disponibles para analizar un caso; en lo que sigue se revisará brevemente cada uno de estos aspectos.

La bioética contemporánea no está libre de disputas y controversias, que tienen su origen en la coexistencia de distintas corrientes de fundamentación ética ${ }^{(13)}$, las cuales difieren en el modo de calificar la bondad o maldad de las acciones humanas (Tabla 1). Si bien los $\mathrm{CEH}$ no declaran formalmente su cercanía o lejanía con alguna corriente ética, es indudable que sus sugerencias están sustentadas en los principios, valores y creencias de sus integrantes, siendo también relevante el marco valórico de la institución a la que pertenecen y el contexto cultural del país o región.

Tabla 1. Principales tendencias en la bioética contemporánea

\begin{tabular}{|l|}
\hline - Tradición hipocrática \\
\hline - Utilitarismo \\
\hline - Libertarismo \\
\hline - Ética kantiana \\
\hline - Casuística \\
\hline - Ética relacional y feminista \\
\hline - Ética de la virtud \\
\hline - Principialismo \\
\hline - Personalismo \\
\hline
\end{tabular}

Tomado y modificado de ${ }^{(13)}$.

En la sistematización del análisis se han reconocido diversos modelos, entre los cuales destacan los norma- 
tivos y los descriptivos ${ }^{(14)}$. De ellos, los más utilizados son los modelos normativos, que surgen de la moral tradicional, y que consisten en la aplicación de una corriente de fundamentación determinada a los casos de la vida cotidiana. Estos modelos tienen una primera fase descriptiva, en la que se recopilan datos sobre el problema a analizar; seguida de una fase de juicio, en la que se aplican las normas o un sistema de fundamentación ética a la situación particular.

Por último, importa utilizar una metodología para analizar de manera sistemática cada caso ético-clínico. En Latinoamérica se conoce el modelo deliberativo, propuesto por Diego Gracia, con sus 3 fases deliberativas: sobre los hechos, sobre los valores y sobre los deberes ${ }^{(15)}$. Un método alternativo se desarrolló por Paulina Taboada, en la Universidad Católica de Chile (Tabla 2), e incluye 8 pasos, que se inician con la identificación del problema ético (formulado por medio de una pregunta), el reconocimiento y ponderación de los principios involucrados, la información clínica éticamente relevante y la participación del paciente o su familia. Con estos elementos se analizan las distintas alternativas de acción y se elige una de ellas, la que pasa a sugerirse como solución del problema. La última etapa consiste en definir la implementación práctica de la alternativa elegida y reflexionar acerca de los aprendizajes del caso ${ }^{(16)}$.

Tabla 2. Sistematización del análisis ético de casos clínicos

\begin{tabular}{|l|}
\hline $\begin{array}{l}\text { 1. Identificar el(los) problema(s) ético(s), y formular } \\
\text { operacionalmente la(s) pregunta(s) }\end{array}$ \\
\hline 2. Revisar los valores o principios éticos involucrados \\
\hline $\begin{array}{l}\text { 3. Recolectar y analizar la información clínica éticamente } \\
\text { relevante }\end{array}$ \\
\hline $\begin{array}{l}\text { 4. Evaluar la capacidad del paciente de participar en la toma } \\
\text { de decisiones; tomar en cuenta sus valores o preferencias }\end{array}$ \\
\hline 5. Revisar las posibles alternativas de acción \\
\hline 6. Formular la respuesta a la pregunta ética \\
\hline 7. Evaluar la mejor forma de implementar la solución \\
\hline 8. Reflexionar sobre los aprendizajes del caso \\
\hline
\end{tabular}

Tomada y modificada de ${ }^{(16)}$.

\section{DILEMAS ÉTICOS EN LA NUTRICIÓN}

\section{Consideraciones preliminares}

La nutrición e hidratación son fundamentales para sostener la vida y permitir el crecimiento, desarrollo y reproducción de los seres vivos. En los seres humanos, alimentarse no solo cumple este rol fisiológico, sino que es parte integral de la vida en sociedad, con un fuerte significado cultural y religioso, dependiendo de las etnias y zonas geográficas que se consideren ${ }^{(17)}$.

Se crece compartiendo el alimento en la familia; luego la comida y la bebida siguen presentes en los eventos más relevantes de la vida en comunidad. Del mismo modo, el proporcionar agua y alimento al necesitado se ha considerado una obligación en todas las épocas y culturas, deber que emana de la caridad y constituye una manifestación de hospitalidad y acogimiento entre las personas. Por lo mismo, en general nadie se cuestiona el deber básico de alimentar a quienes lo necesitan, y se considera reprochable el desperdicio de alimentos en un mundo en el que aún existen grupos humanos que sufren hambre y desnutrición.

En la atención de salud, los aspectos médico-nutricionales (que incluyen suplementación, adaptación de la dieta y nutrición administrada artificialmente) son parte relevante del plan terapéutico y, como tales, requieren una prescripción médica y el consentimiento del paciente. Más aún, existen muchas condiciones clínicas, transitorias o permanentes, en las que los pacientes ya no pueden alimentarse por sí mismos, y se requiere administrar nutrientes, ya sea por el tubo digestivo, utilizando sondas o, en casos en que esto no sea posible, por vía intravenosa.

Es en este contexto que la nutrición e hidratación pasan a denominarse «artificiales», en contraposición a la nutrición natural, y, en determinadas situaciones, dan origen a cuestionamientos respecto a la licitud ética de su prescripción y administración. A nivel social, este tema se actualiza cada vez que se conocen, por los medios de comunicación social, casos emblemáticos de pacientes con grave daño neurológico, en los que se plantea el dilema ético de suspender la nutrición e hidratación artificial para permitir o, según la perspectiva de análisis, causar la muerte.

\section{Comités de ética hospitalaria y los dilemas nutricionales}

La nutrición es un aspecto básico del plan terapéutico de los pacientes hospitalizados y, como se ha dicho, puede dar origen a dilemas éticos. Las publicaciones que reportan la experiencia de los $\mathrm{CEH}$, la mayoría revisiones retrospectivas, no permiten establecer cuán frecuentemente se consultan dichos comités por cuestiones relacionadas con la nutrición ${ }^{(18)}$. Quizás esto se 
explica porque utilizan clasificaciones más amplias de las intervenciones que generan dilemas éticos, como «terapias de soporte vital» ${ }^{(19)}$.

Del análisis de los datos publicados se concluye que la mayoría de las consultas proviene de situaciones que afectan a pacientes críticos en los extremos de la vida, y se refieren a dos ámbitos predominantes: en primer lugar, los comités se consultan por decisiones de adecuación o limitación del esfuerzo terapéutico; en segundo lugar, por dilemas generados por discordancias entre los deseos del paciente (o de su familia o representante) y la propuesta de los médicos, siendo lo más frecuente el rechazo de terapias.

Con respecto a la adecuación del esfuerzo terapéutico, los médicos consultan por la licitud ética de no iniciar o suspender medidas de soporte vital (witholding or withdrowing treatments), tales como reanimación cardiopulmonar, ventilación mecánica invasiva, terapias de soporte renal o intervenciones quirúrgicas mayores, por mencionar las más relevantes. Entre estas intervenciones que sostienen la vida también se incluye la nutrición e hidratación administrada artificialmente.

Para el análisis de este tipo de dilema ético, junto con la consideración de los principios tradicionales de no maleficencia, beneficencia, autonomía y justicia, es de ayuda recurrir al principio de proporcionalidad terapéutica. Este antiguo principio de la tradición moral establece que las medidas que guarden una relación de debida proporción entre el esfuerzo de su implementación y el resultado esperable son proporcionadas y, por tanto, obligatorias ${ }^{(13)}$. Es así como al realizar un adecuado juicio de proporcionalidad es posible diferenciar, para cada caso particular, terapias moralmente obligatorias (proporcionadas) de aquellas que no lo son (desproporcionadas). De allí surge la habitual recomendación de los $\mathrm{CEH}$ de no implementar tratamientos desproporcionados, lo que evita el empecinamiento terapéutico y permite la muerte, si es el caso. Obviamente, el comité recuerda a los consultantes el carácter obligatorio de las terapias proporcionadas.

En este sentido, la alimentación por la vía oral nunca se cuestiona, por ser una medida básica y obligatoria, junto con las medidas de alivio sintomático y cuidados generales. No ocurre lo mismo con la nutrición e hidratación administrada artificialmente (NHAA), ya sea por medio de sondas enterales o por vía intravenosa, la cual es causa de frecuentes cuestionamientos éticos, como es el caso de su prescripción en pacientes con demencia avanzada, daño neurológico grave e irrecuperable, cáncer en etapa terminal y en moribundos. En algunos de estos contextos clínicos, la nutrición parenteral total central, que implica mayores cargas, costos y riesgos, se considera una medida desproporcionada o fútil, en especial, cuando para ese mismo paciente se ha sugerido no implementar o suspender otras terapias de soporte vital.

La segunda causa de problemas ético-clínicos analizados en los $\mathrm{CEH}$ es el rechazo de terapias o la situación opuesta, es decir, la solicitud de insistir en el esfuerzo terapéutico. El rechazo de la propuesta terapéutica se considera un derecho del paciente, ya que nadie podría ser sometido a intervenciones médicas en contra de su voluntad, salvo excepciones relacionadas con la salud pública o el bienestar de terceras personas. En estos casos, el comité debe asegurarse de que el paciente tenga la capacidad para decidir, haya sido informado adecuadamente de la naturaleza del tratamiento y comprenda las consecuencias de su rechazo; finalmente, que la decisión haya sido tomada con libertad, es decir, en ausencia de coacción o coerción. Si el paciente se encuentra incapacitado para decidir, como menores de edad, pacientes inconscientes o con demencia avanzada, se debe velar por que quienes representan su voluntad lo hagan en su mejor interés o bien integral.

En los países que se encuentran reguladas las voluntades anticipadas, se debería tener presente esta manifestación de voluntad del paciente. Por el contrario, la solicitud de realizar nuevas terapias o insistir en el esfuerzo terapéutico en contra de la voluntad del equipo sanitario no tiene carácter vinculante, ya que siendo el médico el agente moral, es el único responsable de sus acciones y no está obligado a actuar en contra de sus convicciones profesionales.

En los siguientes párrafos, y a modo de ejemplo, se expondrán brevemente tres dilemas ético-clínicos relacionados con la nutrición, y que podrían ser motivo de consulta a los $\mathrm{CEH}$ : la solicitud de alta en pacientes con desnutrición grave por trastornos conductuales, la NHAA en pacientes con demencia avanzada y la NHAA en pacientes terminales.

Existen en la literatura varias revisiones que abordan en mayor profundidad estos temas ${ }^{(20-2)}$; del mismo modo, se pueden consultar los pronunciamientos de la European Society for Parenteral and Enteral Nutrition $(\text { ESPEN })^{(23)}$ y la American Society for Parenteral and Enteral Nutrition (ASPEN) ${ }^{(24)}$. Otras situaciones quedarán sin abordar, como es el caso de la realimentación forzada de pacientes en huelga de hambre o la licitud de brindar cuidados paliativos a pacientes que han decidido dejar de comer y beber para provocarse la muerte (voluntary stopping of eating and drinking), pro- 
blemas que por su complejidad escapan a los objetivos de este artículo. El caso de la suspensión de la NHAA de pacientes en estado de mínima conciencia ${ }^{(22)}$ o en estado vegetativo persistente crónico se abordará en otro capítulo de este número de la revista.

\section{Trastornos de la conducta alimentaria: el caso de la anorexia nerviosa}

Con cierta frecuencia ingresan al hospital general pacientes con grave compromiso nutricional, secundario a trastorno de la conducta alimentaria, como es el caso de la anorexia nerviosa, lo cual plantea una serie de cuestionamientos éticos ${ }^{(25)}$ y legales ${ }^{(26)}$. Por el alto riesgo vital, estos pacientes requieren ser realimentados, a veces de manera coercitiva, gradualmente y bajo estricta monitorización, para evitar los trastornos hidroelectrolíticos, la ingesta de medicamentos no prescritos, el descarte de alimentos o los vómitos autoinducidos. Algunos de estos pacientes solicitan el alta hospitalaria (o se niegan a ser trasladados a clínicas especializadas en estas condiciones) antes de alcanzar un índice de masa corporal «seguro», lo que genera una perplejidad en los médicos tratantes. El CEH debe analizar este problema ético-clínico, valorando adecuadamente el riesgo vital (principio de defensa y cuidado de la vida) frente a la legitimidad de rechazar un plan terapéutico (principio de respeto a la autonomía). La deliberación es más compleja cuando se trata de menores de edad y son sus padres o tutores legales quienes solicitan el alta al domicilio.

\section{NHAA en el paciente con demencia avanzada}

La demencia avanzada es una condición clínica, que se acompaña de incapacidad para autoalimentarse, disfagia, trastornos de la deglución y aspiración broncopulmonar. Los pacientes evolucionan a un compromiso nutricional progresivo, postración, úlceras por decúbito y mayor incidencia de infecciones respiratorias (neumonías aspirativas).

La decisión de suministrar alimentación por medio de una sonda nasogástrica, o más frecuentemente por sonda de gastrostomía, constituye un dilema ético, que puede analizarse en el CEH intentando responder, inicialmente, dos preguntas: una relacionada con la real utilidad de la NHAA en estos pacientes, y en segundo lugar indagando si existe una voluntad previa. Adicionalmente deben considerarse las dificultades, costos y cargas que implica sostener este tipo de nutrición en el tiempo, lo que puede variar de un paciente a otro.
La evidencia disponible en la actualidad permite afirmar que en demencia avanzada, la NHAA no logra mejorar significativamente el estado nutricional, disminuir la incidencia de neumonías por aspiración o las úlceras por decúbito ${ }^{(27)}$. De ello se sigue que no sería una medida obligatoria la instalación de una gastrostomía de alimentación, aunque podría ser opcional en aquellas situaciones en que es bien tolerada por el paciente y disminuye la carga a sus cuidadores. La otra consideración relevante es conocer la voluntad del paciente, aunque no conste por escrito y no se encuentre regulada legalmente en algunos países. Si consta una manifestación reiterada y contraria a ser alimentado por medio de sondas en las etapas finales de la enfermedad, esta voluntad debería considerarse en la toma de decisiones por respeto a la dignidad de la persona, ya que en cierto modo refleja la aceptación del carácter progresivo de la enfermedad y el advenimiento de la muerte natural.

\section{NHAA en el enfermo terminal}

El enfermo terminal es aquel que tiene una enfermedad o condición bien diagnosticada, sin tratamiento curativo o que modifique significativamente su pronóstico, de curso progresivo y que provocará la muerte en un plazo relativamente breve, de algunas semanas a pocos meses ${ }^{(28)}$. Es relevante diferenciar esta condición de otras enfermedades crónicas que, si bien provocan grados de dependencia y postración, no conducen a una muerte próxima. En este último caso se trata de pacientes que con los cuidados básicos y la nutrición pueden vivir largos períodos en condiciones relativamente estables; en cambio, cuando se vive en las proximidades de la muerte, cobran relevancia los cuidados paliativos y surge el cuestionamiento sobre los beneficios de la hidratación y nutrición.

Existe suficiente evidencia en la literatura para afirmar que los pacientes moribundos tienen menos apetito y sed, al tiempo que experimentan alivio por medio de un cierto grado de deshidratación (disminución de los edemas, la tos, las secreciones bronquiales, las manifestaciones digestivas y la necesidad de cateterismos urinarios). En ellos, la sensación de sed se alivia al humedecer los labios y mantener una buena higiene de la cavidad oral ${ }^{(20)}$. La decisión de no insistir en la NHAA será necesariamente prudencial, y deberá considerar el pronóstico de sobrevida, el nivel de conciencia y el grado de dificultad para administrar agua y alimentos (disponibilidad de vías de acceso y tolerancia digestiva, por ejemplo). 
Los $\mathrm{CEH}$ pueden recibir consultas por este dilema ético-clínico. En oportunidades es la familia la que se niega a suspender la NHAA, interpretando esta acción como abandono del paciente o aceleramiento de la muerte. En estos casos, la sugerencia principal del comité es lograr una comunicación efectiva con la familia, y aportar información adecuada respecto a los objetivos de la terapia paliativa, que tiene como fin evitar el sufrimiento innecesario y permitir la muerte en paz.

\section{CONCLUSIÓN}

Transcurridas 6 décadas desde la introducción de los comités de ética hospitalaria es posible afirmar que en la actualidad son una instancia consultiva relevante en el quehacer clínico, que contribuye a una mejor toma de decisiones en los casos complejos. Entre las múltiples causas de consulta se incluyen los dilemas éticos relacionados con la nutrición e hidratación administrada artificialmente, particularmente al final de la vida, ya sea en pacientes con demencia avanzada o en cuidados paliativos. En esta compleja deliberación, los $\mathrm{CEH}$ deben considerar aspectos éticos y legales, así como el valor simbólico y cultural que tiene la alimentación para los seres humanos.

\section{Agradecimientos}

A la Dra. Paulina Taboada Rodríguez por autorizar el uso adaptado de las Tablas 1 y 2 de sendas publicaciones de su autoría.

\section{Financiación}

El presente estudio no tuvo financiación.

\section{Conflicto de intereses}

El autor declara no tener conflicto de intereses.

\section{Declaración de relevancia clínica}

El artículo destaca el rol y la importancia de los comités de ética hospitalaria como instancias de ayuda en la toma de decisiones clínicas complejas, que involucran aspectos éticos. Entre estos se encuentran los dilemas relacionados con la nutrición e hidratación administrada artificialmente.

\section{Declaración de autoría}

I. Pérez es autor de la concepción, diseño, redacción y aprobación de este manuscrito. Declara ser responsable de la integridad y precisión de este trabajo.

\section{Referencias bibliográficas}

1. Bascuñán RML. Cambios en la relación médico-paciente y nivel de satisfacción de los médicos. Rev Med Chile. 2005;133:11-6. doi: 10.4067/S0034-98872005000100002

2. Zuger A. Dissatisfaction with medical practice. N Engl J Med. 2004;350(1):69-75. doi: 10.1056/NEJMsr031703

3. Ley 20584. Regula los derechos y deberes que tienen las personas en relación con acciones vinculadas a su atención de salud [Internet]. Ministerio de Salud, Subsecretaria de Salud Pública, Gobierno de Chile. Consultado el 30 de junio 2021. Disponible en: https://www.bcn.cl/leychile/navegar?idNo $\mathrm{rma}=1039348 \&$ idVersion $=2021-05-11$ \&idParte $=10042018$

4. Tealdi JC, Mainetti JA. Los comités hospitalarios de ética. Bol Oficina Sanit Panam. 1990;108(5-6):431-8.

5. Burmester M. Organización y rol del comité de ética en el hospital general. En: Correa DE, Echeverría BC, Rojas OA (editores). Ética y humanidad en la medicina actual. $1^{\text {a }}$ edición. Santiago de Chile: Editorial Universitaria. 1993. p. 227-37.

6. Lo B, Schroeder SA. Frequency of ethical dilemmas in a medical inpatient service. Arch Intern Med. 1981;141(8):1062-4. doi: 10.1001/archinte.141.8.1062

7. Couceiro VA, Beca IJP. Los comités de ética asistencial y las repercusiones jurídicas de sus informes. Rev Med Chile. 2006;134:517-9. doi: 10.4067/S0034-98872006000400016

8. Funcionamiento de los comités de bioética: procedimientos y políticas [Internet]. Organización de las Naciones Unidas para la Educación, la Ciencia y la Cultura. Paris, Francia: UNESCO. 2006. Consultado el 30 de junio 2021. Disponible en: https://www.cibir.es/files/biblioteca/2006-UNESCOGuia-2-funcionamiento-de-los-comites-de-bioetica.pdf

9. Courtwright A, Jurchak M. The evolution of American hospital ethics committees: A systematic review. J Clin Ethics. 2016;27(4):322-40.

10. Gaudine A, Thorne L, LeFort SM, Lamb M. Evolution of hospital clinical ethics committees in Canada. J Med Ethics. 2010;36(3):132-7. doi: 10.1136/jme.2009.032607

11. Beca IJP, Koppmann AA, Chávez BP, Delgado BI, Solar PS. Análisis de una experiencia de consultoría ético clínica en cuidado intensivo. Rev Med Chile. 2010;138:815-20. doi: 10.4067/S0034-98872010000700004

12. Couceiro A. Comités de ética o consultores de ética ¿qué es lo mejor para las instituciones sanitarias? Bioética \& Debat. 2008;14(54):16-21. 
13. Taboada P. Bioethical principles in palliative care. En: Bruera E, Higginson IJ, von Gurten CF, Morita T (editores). Textbook of palliative medicine and supportive care. $2^{\text {a }}$ edición. Londres: CRC Press. 2015. p. 105-18.

14. López BR. Modelos de análisis de casos en ética clínica. Acta Bioeth. 2015;21(2):281-90. doi: 10.4067/S1726569X2015000200014

15. Gracia D. La deliberación moral: el método de la ética clínica. Med Clín. 2001;117(1):18-23.

16. Taboada P. Decisiones al final de la vida. En: Andresen HM (editor). Manual de medicina intensiva. $3^{\mathrm{a}}$ edición. Santiago de Chile: Editorial Mediterráneo. 2019. p. 457-65.

17. Martin FC, Abyad A, Arai H, Arcand M, Hasan BH, Beattie $\mathrm{BL}$, et al. Eating and drinking in later life. En: Michel JP, Beattie BL, Martin FC, Walston J (editores). Oxford textbook of geriatric medicine. $3^{\text {a }}$ edición. Oxford University Press. 2017. p. 1213-27.

18. Tapper EB, Vercler CJ, Cruze D, Sexon W. ethics consultation at a large urban public teaching hospital. Mayo Clin Proc. 2010;85(5):433-8. doi: 10.4065/mcp.2009.0324

19. Wasson K, Anderson E, Hagstrom E, McCarthy M, Parsi K, Kuczewski $\mathrm{M}$. What ethical issues really arise in practice in an academic medical center? A quantitative and qualitative analysis of clinical ethics consultations from 2008 to 2013. HEC Forum. 2016;28(3):217-28. doi: 10.1007/s10730-0159293-5

20. Geppert CMA, Andrews MR, Druyan ME. Ethical issues in artificial nutrition and hydration: A review. JPEN J Parenter Enteral Nutr. 2010;34(1):79-88. doi: $10.1177 / 0148607109347209$
21. Cárdenas D. Ethical issues and dilemmas in artificial nutrition and hydration. Clin Nutr ESPEN. 2021;41:23-9. doi: 10.1016/j.clnesp.2020.12.010

22. Newcomer KF, Fine RL, Newman AF. Food as love: Ethical and moral dilemmas in withdrawal of artificial nutrition and hydration in the minimally conscious state. J Palliat Care. 2021. doi: $10.1177 / 08258597211014359$

23. Druml C, Ballmer PE, Druml W, Oehmichen F, Shenkin A, Singer P, et al. ESPEN guideline on ethical aspects of artificial nutrition and hydration. Clin Nutr. 2016;35(3):545-56. doi: 10.1016/j.clnu.2016.02.006

24. Schwartz DB, Barrocas A, Annetta MG, Stratton K, McGinnis C, Hardy G, et al. Ethical aspects of artificially administered nutrition and hydration: An ASPEN position paper. Nutr Clin Pract. 2021;36(2):254-67. doi: 10.1002/ncp.10633

25. Figueroa G. Bioética de la anorexia nerviosa ¿autonomía, beneficencia o responsabilidad? Rev Med Chile. 2008;136:659-65. doi: 10.4067/S0034-98872008000500017

26. Carney $\mathrm{T}$, Tait $\mathrm{D}$, Wakefield $\mathrm{A}$, Ingvarson $\mathrm{M}$, Touyz $\mathrm{S}$. Coercion in the treatment of anorexia nervosa. Clinical, ethical and legal implications. Med Law. 2005;24(1):21-40.

27. Sampson EL, Candy B, Jones L. Enteral tube feeding for older people with advanced dementia. Cochrane Database Syst Rev. 2009;2009(2):CD007209. doi: 10.1002/14651858. CD007209.pub2

28. Grupo de Estudios de Ética Clínica de la Sociedad Médica de Santiago. El enfermo terminal. Rev Med Chile. 2000;128(5):547-52. doi: 10.4067/S003498872000000500015 